\title{
La Epistemología en la Ingeniería Agroindustrial
}

\author{
The Epistemology in Agroindustrial Engineering \\ ${ }^{1}$ Gino Paul Prieto Rosales ${ }^{\mathrm{a}},{ }^{2}$ Lucia Ruth Pantoja Tirado ${ }^{\mathrm{a}}$
}

\section{RESUMEN}

La epistemología en la ingeniería agroindustrial representa una disciplina Filosófica que toma su autonomía en particular desde distintas corrientes del siglo XVII. El campo de acción de esta disciplina ha versado esencialmente sobre la posibilidad de representación de la inteligencia humana. El objetivo principal de este trabajo es brindar a los profesionales y estudiantes de la carrera de ingeniería agroindustrial un material que les permita entender las bases filosóficas de la ciencia aplicada en los productos alimentarios y no alimentarios, obtenidos a través de materias primas de origen biológico; manipulados para su adecuación, transformación, conservación y comercialización. El trabajo fue desarrollado mediante la revisión exhaustiva de la bibliografía reciente disponible, haciendo énfasis en la filosofía agroindustrial y sus principales ramas y en los enfoques lógico-positivista, hipotéticodeductivo, paradigmático y cibernético-sistémico de la ciencia. En general, se puede afirmar que la epistemología es aquella doctrina sobre los fundamentos del conocer de la ciencia o los modos o perspectivas a través de las cuales procede dicho conocer, lo cual cubre todas aquellas áreas de las ciencias que se basan en el método científico, que poseen un área del conocimiento propio y que cuentan con el consenso de la comunidad científica.

Palabras clave: epistemología, ciencia, ingeniería agroindustrial, filosofía agroindustrial.

\begin{abstract}
In the agroindustrial engineering epistemology represents a philosophical discipline that takes its autonomy in particular from different currents of the XVII century. This discipline has been studied essentially about the possibility of representation of human intelligence. The main objective of this work is to provide both, professionals and students of the agroindustrial engineering career with material that allows them to understand the philosophical bases of applied science in food and non-food products, obtained through of biological raw materials; manipulated for its adaptation, transformation, conservation and commercialization. This work was developed through the exhaustive revision of the available recent bibliography and emphasis was made in the agroindustrial philosophy and its main branches and in the logical-positivist, hypothetic-deductive, paradigmatic and cybernetic-systemic approaches of the science. In general, it can be said that epistemology is that doctrine about the foundations of the knowledge of science or the modes or perspectives through which this knowledge proceeds, which covers all those areas of science that are
\end{abstract}


based on the scientific method, that have both, an area of their own knowledge and the consensus of the scientific community.

Key words: epistemology, science, agroindustrial engineering, agroindustrial philosophy.

${ }^{1}$ Universidad Nacional Autónoma de Tayacaja Daniel Hernández Morillo

${ }^{a}$ Ing. Agroindustrial 


\section{INTRODUCCIÓN}

La epistemología se denomina como la disciplina que tiene por objeto de estudio el conocimiento y se constituye como una rama dentro de la filosofía. En este sentido, aborda de qué manera se logra la obtención del conocimiento, así como también las circunstancias en las cuales se obtiene, ya sean éstas sociológicas, históricas, entre otras. La epistemología, al igual que la filosofía, comenzó a desarrollarse en la antigüedad, cuando los primeros filósofos griegos, como Parménides, iniciaron las primeras reflexiones acerca de cómo el hombre obtiene conocimiento. Castillo (2015).

Dentro del amplio mundo de la epistemología existe un marco muy interesante e importante, este se conoce como estatuto epistemológico, el cual puede ser definido como aquella doctrina sobre los fundamentos del conocer de la ciencia; por otra, los modos o perspectivas a través de las cuales procede dicho conocer. Tienen este estatuto las ciencias que se basan en el método científico, que poseen un área del conocimiento propio y que cuentan con el consenso de la comunidad científica. Las ciencias sociales no han resuelto a plenitud su estatuto epistemológico, pero tampoco podemos esperar que se estructuren a semejanza de las ciencias duras, que tienen otros objetos de estudio y no están enfrentadas a la complejidad humana.

En conclusión, un estatuto epistemológico estudia la producción y validación de un conocimiento científico, dando prioridad a este (ciencia de estudio). Castillo (2015). Según el estatuto epistemológico una ciencia para que sea válida de debe poseer:

- Un campo de estudio que sea propio.

- Un cuerpo de contenidos validados.

- Un lenguaje propio.

- Autonomía metodológica.

\section{La realidad y sus diferentes escenarios}

La Ingeniería Agroindustrial es la rama de la ingeniería de realidad natural, basándose en las Ciencias de la Matemática, Ciencias Naturales, ciencias cruzadas como la Bioquímica, Biotecnología, Ciencias Económicas, Administrativas, y Ciencias de la Ingeniería, las aplica tanto a los procesos productivos como de gestión en la agroindustria, sean estas dedicadas a procesos tanto alimentarios como no alimentarios. Castillo (2015).

\section{Percepción de la realidad exterior}

Nuestra percepción de la realidad de la Ingeniería Agroindustrial es el fruto de lo que viene de nuestros sentidos, la secuencia seria: (Figura $\mathrm{N}^{\circ} 1$ )

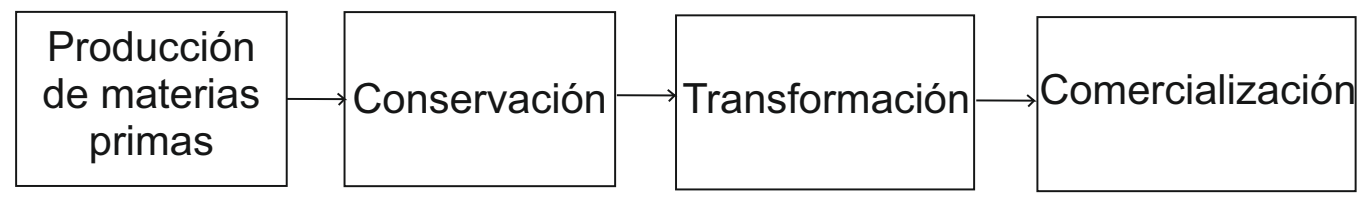

Figura $\mathrm{N}^{\circ} 01 .-$ Secuencia de la Ingeniería Agroindustrial 


\section{La Ingeniería Agroindustrial tiene} como función la de relacionar la agricultura y la ganadería con la industria, su materia prima es la producción agropecuaria que a través de procesos industriales es transformada en productos con valor agregado, como bebidas, embutidos, aceites, tejidos, perfumes y enlatados, con fines alimentarios y no alimentarios, bajo criterios de sostenibilidad. Vejarano (2015).

\section{Conocimiento de la realidad}

La Ingeniería Agroindustrial aplica la ciencia, ingeniería y tecnología agroindustrial a la investigación, diseño, desarrollo, operación y control de los equipos y procesos en las fases de producción, industrialización y comercialización de los productos finales del agro a precios competitivos en el mercado de consumo.

\section{La filosofía agroindustrial y sus} principales ramas

Los principios del Ingeniero Agroindustrial son "creatividad y aportación". Son profesionales competentes, responsables y útiles, que proveen una aportación social a la sociedad, en un ambiente armónico de respeto, colaboración y confianza, sustentado en una sólida organización que además impulsa la incorporación de los avances de la ciencia, la investigación, la aplicación del conocimiento, la innovación educativa, la evaluación, la calidad y el intercambio cultural, todo dentro de un marco de sustentabilidad. Domínguez (2019).

\section{La ciencia actual y su estructura}

\section{Identificación del objeto de estudio}

La Ingeniería Agroindustrial, objeto de estudio, se enfatiza en los productos alimentarios y no alimentarios, obtenidos a través de materias primas de origen biológico; manipulados para su a d e cuación, transformación, conservación y comercialización. Vejarano(2015).

\section{La actividad científica}

Si nos referimos al método existente en la Ingeniería Agroindustrial, es una subdivisión de la ingeniería, la cual maneja como método principal el hipotético deductivo, que consiste en hacer observaciones manipulativas $\mathbf{y}$ análisis, a partir de las cuales se formulan hipótesis que serán comprobadas mediante experimentos controlados.

Aunque esta no es la única forma de hacer ciencia, es la más utilizada y validada, además de esto que se repite constantemente, durante el cual se examinan hipótesis a la luz de los datos que van arrojando los experimentos. Si la teoría no se ajusta a los datos, se ha de cambiar la hipótesis, o modificarla, a partir de inducciones. Castillo (2015).

\section{Las primeras etapas del racionalismo y empirismo y del desarrollo de la ciencia}

El rasgo común de lo que se denomina ciencia es la racionalidad como principio 
epistemológico puro; la racionalidad, a su vez, es inherente a la naturaleza misma del sistema capitalista que se consolida como tal en el siglo XVIII. Para imponerse, los científicos lucharon frontalmente contra el predominio del principio teológico que constituía el orden feudal, al tiempo que convivieron, desde el renacimiento en el siglo XV y XVI, con la filosofía, la religión, la masonería, la astrología, la metafísica, lo esotérico, hasta que desde propuestas individuales y luego con carácter social o de tendencia social, pudo constituirse, la ciencia, como la única vía de explicación de la realidad natural, en el caso de las ciencias naturales, y de la realidad social, en el caso de las ciencias sociales. Méndez (2000).

La racionalidad pura, generada y aplicada por si misma por el hombre intelectual o científico, en un esfuerzo de concentración mental sobre la realidad, que explica con categorías, conceptos y teorías especificas dicha realidad, elimina el animismo, las esencias o las influencias planetarias, así como también, la suerte y la casualidad. Esta racionalidad se socializó, inclusive para todos los individuos de la sociedad, vía escuela, al convertirse la ciencia en el único saber que proporciona la verdad. Ciencia y verdad absoluta eran lo mismo. Esto va sucediendo desde el mismo momento en que empieza a emerger el capitalismo, como sistema social universal, hegemónico y concreto. Durante su nacimiento y desarrollo se buscaron respuesta a los nuevos problemas y necesidades del hombre. Recuérdese las hambrunas, la peste, el aumento de la población, la aparición de las ciudades, el mercado, la constitución de las naciones, el descubrimiento de América, el comercio por occidente, los nuevos ejes de poder en Europa. Pero esto sólo es posible si la racionalidad se imponía a través de la ciencia y la técnica. La idea era analogar racionalidad y ciencia. De modo que quienes lideraron el capitalismo, asumieron la ciencia y la técnica como medio para imponer y consolidar el nuevo régimen social, y para mantenerse y perpetuarse en el poder.

Intelectuales como Descartes sistematizan la ciencia moderna, desde la razón y la pureza, donde la objetividad era la carta que garantizaba la aplicación del método científico. Descartes establecía que dedicaría toda la vida a "cultivar la razón siguiendo el método que se había propuesto". Todo esto para lograr la autonomía de la ciencia. El programa de Descartes, concretado en la expresión "Pienso y luego existo" (Descartes), constituye la tesis central del racionalismo moderno, unido a las reglas del método y la duda que se tiene previamente acerca del saber acumulado. Este planteamiento se opuso aparentemente al planteamiento de Galileo quien introdujo el criterio experimental en la ciencia moderna. Igualmente, a Francis Bacon en Inglaterra quien fundamentó la ciencia a partir del empirismo. 
Este postulaba que el origen del conocimiento estaba en la experiencia del investigador y la esencia última del conocimiento estaba en el objeto de estudio, por ello el método científico debe ser el inductivo. Hay que comenzar la investigación por la observación, luego en base a la regularidad que presentan las variables del problema, se formula una hipótesis que deberá verificarse experimentalmente; de ser así y utilizando la inferencia inductiva se formula la teoría que explica en forma universal el problema de estudio; esta posición se fortalece con el empirismo que sistematiza Locke. Es asombroso cómo hoy existen autores de manuales de metodología que todavía promueven este procedimiento, por ejemplo, Neil Salkind es uno de ellos.

El Darwinismo en el siglo XIX, consolida la modernidad al descartar el origen divino del hombre, al insertarlo en su teoría evolucionista, según la cual las especies para sobrevivir deben ser las más aptas, y asombrosamente se complementa el proyecto de la modernidad ya desde finales del siglo XVIII y durante el XIX, con los opositores al régimen social, que asumen inclusive la racionalidad absoluta como única vía. Marx y Engels son un ejemplo de ello, cuestión que es emulada por todos los planteamientos socialistas y comunistas posteriores.

La racionalidad científica desde que se planteó no ha variado a nivel de las instituciones formales de la sociedad que auspician el desarrollo de este conocimiento, como es el caso de universidades, colegios, asociaciones, y editoriales, cuestión que encierra un peligro inminente para el III milenio, y que explicaremos más adelante. Se materializa la racionalidad como paradigma, como explica Kuhn en su libro "La Estructura de las Revoluciones Científicas". Es decir, no sólo se fundamenta intelectualmente la ciencia, sino que se legitima en el seno de las comunidades científicas y en toda la sociedad. En las comunidades científicas se termina abordando siempre de la misma manera todos los problemas que la realidad plantea, generándose círculos viciosos al reproducir el conocimiento trayendo esto como consecuencia la paralización del avance científico. Si se aplica una metodología sin cuestionamiento epistemológico se asemeja a un dogma que no se diferencia de los mitos religiosos que ha tenido el hombre.

En la modernidad se trató de vender la filosofía como especulación, como metafísica. La único que se admitió como filosofía, fue entonces la llamada filosofía de la ciencia o epistemología, colocando en forma secundaria y hasta eliminando la discusión en torno a la ontología y la axiología. Autores como Descartes, Kant, y Hegel son un claro ejemplo de ello. En forma marginal y no oficial, el marxismo en el siglo XIX, la fenomenología, y el existencialismo en el siglo XX, restablecían el carácter ontológico y axiológico de la filosofía, pero por su 
carácter ideológico político permanecieron en una posición secundaria. Al abandonar la filosofía, en el orden institucional científico se le sometió a una especie de operación y le extrajeron la episteme, que traducida quiere decir ciencia y significaba para entonces el conocimiento racional por causas, y este principio lo introdujeron inicialmente en las ciencias particulares. Desde entonces, el desarrollo de la ciencia oficial ha estado en torno a la lógica del conocimiento. Se estableció una polémica entre el racionalismo y el empirismo, entre la inducción y la deducción, entre la verificación y la refutación. Entre el argumento o el dato. Locke, Hume, Kant, y luego toda la filosofía analítica o el neopositivismo lógico en el siglo XX y el mismo Karl Popper desde 1934, con su racionalismo crítico y sus discípulos ingleses, de fin de siglo, fundamentaron la ciencia sólo desde el punto de vista gnoseológico. Posteriormente, los profesores dogmáticos simplificaron esta polémica a la enseñanza de la metodología de la investigación, como conjunto de pasos que hay que seguir porque de lo contrario al ser violados terminan pagando muy caro el precio: "la investigación resultante no es válida o confiable". Méndez (2000).

\section{LEAN SIX SIGMA EN PEQUEÑAS Y MEDIANAS EMPRESAS: UN ENFOQUE METODOLÓGICO}

Felizzola y Luna (2014). En su investigación sostienen que, Six Sigma y Manufactura Esbelta son enfoques de mejora de la calidad y productividad que han sido implementados con gran éxito en grandes empresas a nivel mundial, en el ámbito de la manufactura y los servicios. Pero en la actualidad investigadores y expertos en el tema han encontrado hallazgos que evidencian dificultades en la implementación de este tipo de enfoques en pequeñas y medianas empresas (PYMES).

Se propone una metodología para la implementación de un enfoque integrado, comúnmente llamado Lean Six Sigma (LSS), el que se adapta a las necesidades y características de las PYMES. La metodología está compuesta de cuatro fases: donde la primera establece los factores claves en los cuales las PYMES deben prepararse para implementar LSS; en segundo lugar, se plantea la identificación de focos de mejora y definición de un portafolio de proyectos; en tercer lugar, la ejecución de los proyectos priorizados; y por último, la evaluación de los resultados obtenidos.

Según Aguilar (2018). El filósofo Adam Smith argumenta que la clave para la riqueza de las naciones es la producción y el intercambio, no la adquisición de oro y plata a expensas de otras naciones. De esta forma descubrió la clave de la prosperidad: libertad económica de la gente. Por otro lado, Frederick Taylor consideró a la productividad como la respuesta al deseo, tanto de salarios más 
altos, como de mayores utilidades. Pensaba que, con la aplicación de métodos científicos, en lugar de la costumbre y métodos prácticos, se podría aumentar la productividad sin gastar más energía o esfuerzo humano.

Por su parte, Henri Fayol identificó 5 funciones de los gerentes: planean, organizan, mandan, coordinan, controlan. El estadístico W. Edwards Deming, fue un gran difusor de la calidad total, estableciendo los siguientes principios:

- Consecución de la plena satisfacción de las necesidades del cliente (interno y externo).

- Desarrollo de un proceso de mejora continua.

- Total compromiso y liderazgo activo de la dirección.

- Participación de todos los miembros de la organización y trabajo en equipo.

- Involucramiento del proveedor en el sistema de calidad total.

- Identificación y gestión de los procesos claves de la organización.

- Toma de decisiones con base a datos y hechos objetivos.

Los filósofos mencionados anteriormente argumentan que para ser competitivos en la empresa se debe tener libertad financiera, aplicar métodos científicos para aumentar la productividad en la empresa y herramientas que permitan mejorar la calidad y la productividad.

En pocas palabras, Six Sigma es un método basado en datos que examina los procesos repetitivos de las empresas y tiene por objetivo llevar la calidad hasta niveles cercanos a la perfección. Es más, se propone una cifra: 3.4 errores o defectos por millón de oportunidades. Se distingue de otros métodos en el hecho de que corrige los problemas antes que se presenten. Felizzola y Luna (2014)

E N F O Q U E S L Ó G I C O POSITIVISTA， HIPOTÉTICODEDUCTIVO, PARADIGMÁTICO Y CIBERNÉTICO-SISTÉMICO DE LA CIENCIA

\section{EL CÍRCULO DE VIENA}

Moritz Schilick inaugura el llamado Circulo de Viena, en los años veinte. Allí se reúnen filósofos y científicos de primera línea, como Carnap, Schlick, Neurath, Feigl, Gödel.

$\mathrm{Su}$ preocupación era utilizar la lógica como herramienta precisa para el análisis de las teorías. Esta escuela se conoce como empirismo lógico o neopositivismo. (Ver figura $\mathrm{N}^{\circ} 2$ )

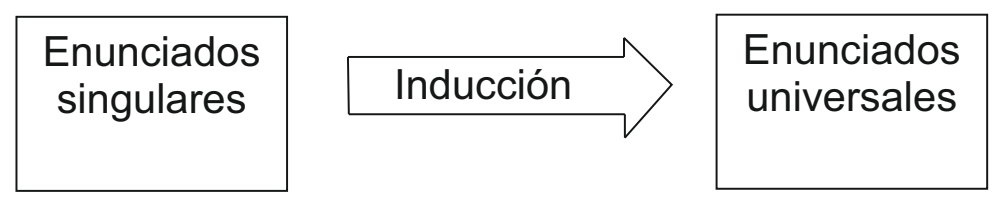

Figura $\mathrm{N}^{\circ} 2$. Circulo de Viena 
¿Bajo qué circunstancias es lícito aseverar que una ley científica ha sido "derivada" de un número finito de pruebas?

Para los inductivistas "Si en un número suficientemente grande de casos se ha dado una relación, esta será válida para todos los casos" Aguilar(2018).

\section{EI problema de la Inducción}

Popper fue contemporáneo y crítico de los miembros del círculo de Viena. Se siente profundamente impresionado por la revolución relativista. Primero, por originalidad y creatividad de las hipótesis planteadas, que la hacían incompatibles con el inductivismo, y segundo porque dejaron al descubierto la falibilidad de cualquier tipo de teoría científica.

En esa época Popper reflexiona acerca de la concepción verificacionista e inductivista de la ciencia, que lo llevan a elaborar el concepto de asimetría entre verificación (imposible) y falsación de las teorías científicas. Aguilar (2018).

\section{La filosofía de la ciencia (Ingeniería Agroindustrial)}

La Ciencia es el intento sistemático de producir proposiciones verdaderas sobre el mundo. O sea que es ese creciente cuerpo de ideas, que puede caracterizarse como conocimiento racional, sistemático, exacto, verificable y por consiguiente falible. La ciencia puede ser considerada como la suma actual de conocimientos científicos, como una actividad de investigación o hasta como un método de adquisición del saber.
La Ingeniería Agroindustrial es la ciencia aplicada que tiene por objeto transformar los productos provenientes del campo sean estos ganaderos o agrícolas en vienes que satisfagan la necesidad del hombre.

La ciencia se confecciona como un sistema de pensamiento absoluto y autónomo que solamente se puede realizar por medio del método científico. Ahora bien ¿qué relación va a tener la ciencia con la filosofía? Aquí ocurrió una profunda tergiversación histórica y teórica. Al pasar la ciencia a ocupar el papel hegemónico, ya no importaba discutir sus fundamentos, por lo que se anuló el papel de la filosofía. Esta, en el mejor de los casos, se redujo en el ámbito oficial, a un discurso de segundo orden, cuya misión consistiría en fundamentar la ciencia. En el peor de los casos, se le relega a un pensamiento etéreo, especulativo, o metafísico. Pero este papel de la filosofía se fundamenta en la lógica y no en la historia, era formal y no de contenido. La tergiversación consistió en negar en primer lugar el origen de la ciencia en la filosofía de la Grecia antigua, y en segundo lugar, el negar la filosofía como ciencia de los principios que buscaba explicar de manera integral la realidad, el hombre y el universo. Lo único que se admitió como filosofía, fue entonces la llamada filosofía de la ciencia o epistemología, colocando en forma secundaria y hasta eliminando la discusión en torno a la ontología y la axiología. Autores como Descartes y Kant, reducen la filosofía al ámbito 
gnoseológico; los demás temas quedaron en una posición marginal. En forma marginal y no oficial el marxismo en el siglo XIX, la fenomenología, y el existencialismo en el siglo XX restablecían el carácter ontológico y axiológico de la filosofía., pero por su carácter ideológico político permanecieron en una posición secundaria y sin ningún impacto en el desarrollo de las instituciones que promueven la ciencia. Aguilar (2018).

La epistemología solo como lógica de la ciencia

Desde entonces, el desarrollo de la ciencia oficial ha estado en torno a la lógica del conocimiento. Se estableció una polémica entre el racionalismo y el empirismo, entre la inducción y la deducción, entre la verificación y la refutación. Entre el argumento o el dato. Locke, Hume, kant, y luego toda la filosofía analítica o el neopositivismo lógico en el siglo XX y el mismo karl Popper desde 1934 con su racionalismo crítico y sus discípulos ingleses, de fin de siglo, fundamentaron la ciencia sólo desde el punto de vista gnoseológico. La epistemología sólo se dedicó a analizar problemas lógicos y metodológicos. Siendo esto muy parcial Y los profesores dogmáticos simplificaron esta polémica a la enseñanza de la metodología de la investigación como conjunto de pasos que hay que seguir porque de lo contrario al ser violados terminan pagando muy caro el precio: "la investigación resultante no es válida o confiable" (Hernández y Otros.1998:
XXVIII). Pero el problema no es reducir la investigación a la metodología, sino que elimina el carácter procesal de la misma, en el sentido de construcción del conocimiento y la sustituyen por la enseñanza de las recetas metodológicas. Son once los pasos para tener éxito en la investigación científica, señalan algunos manuales latinoamericanos de reciente publicación y aceptación en pregrado y postgrado. O son cuatro los capítulos que tiene la investigación científica, estos son el problema, el marco teórico, el marco metodológico y los resultados, según algunos autores y profesores de gran experiencia, los cuales no citaremos para no herir susceptibilidades. En esto cada quien que asuma su responsabilidad. El método concebido de esta manera no es más que un planteamiento vacío, sin contenido. Debe ser entendido como una de las vías para conquistar y construir el conocimiento. El autor de éste trabajo considera que, aun admitiendo cuatro etapas de la ciencia, es necesario fundamentarlas epistemológicamente. Aguilar(2018)

Al asumir la ciencia como pasos seguros que hay que aprenderse trae como consecuencia que muchos jóvenes científicos o aspirantes a obtener títulos de pregrado y postgrado se "parten la cabeza" tratando de realizar ciencia con ese esquema, sin obtener algún resultado positivo. Al darse cuenta de esto, la frustración, la salud y estado físico $\mathrm{y}$ emocional se disparan de manera negativa 
para luego terminar abandonando el trabajo de investigación. O simplemente se les terminó la prórroga que establecen las universidades para terminar y discutir la tesis. Por eso aparece el síndrome "todo menos tesis": TMT. Por ello, en definitiva, no hay pasos, ni fases sino procesos de construcción de conocimiento unido a otros procesos no necesariamente lógicos o racionales como anota Feyerabend (1984) cuando explica el carácter no lógico de la ciencia.

\section{El hipotético-deductivismo (Ingeniería agroindustrial)}

La Ingeniería Agroindustrial posee validación según el estatuto epistemológico ya que, cumple con todos, por decirlo así, sus requisitos (objeto de estudio y su propio método, entre otros). En cuanto al objeto o campo de estudio la ingeniería agroindustrial se enfatiza en los productos alimentarios y no alimentarios, obtenidos a través de materias primas de origen biológico; manipulados para su a d e cuación, transformación, conservación y comercialización. Dicho de otra manera, la Ingeniería Agroindustrial es una rama de la ingeniería, cuyo objeto de estudio es el manejo, conservación, transformación y comercialización de productos agropecuarios, con fines alimentarios y no alimentarios, bajo criterios de sostenibilidad. Castillo (2015).

Si nos referimos al método existente en la Ingeniería Agroindustrial siendo una subdivisión de la ingeniería, esta se maneja como método principal el hipotético deductivo el cual consiste en hacer observaciones manipulativas y análisis, a partir de las cuales se formulan hipótesis que serán comprobadas mediante experimentos controlados.

Aunque esta no es la única forma de hacer ciencia, es la más utilizada y validada, además de esto que se repite constantemente, durante el cual se examinan hipótesis a la luz de los datos que van arrojando los experimentos. Si la teoría no se ajusta a los datos, se ha de cambiar la hipótesis, o modificarla, a partir de inducciones.

Casi simultáneamente, en la década de los años treinta del siglo XX, aparece Karl Popper, (epistemólogo nacido en Viena), cuestionando la lógica inductiva y probabilística y el criterio de verificación como criterio de verdad, y como fundamento de las ciencias empíricas. $\mathrm{La}$ lógica inductiva no tenía criterios para justificar y fundamentar, el pase de lo particular a lo universal, igual pasaba con la probabilidad, ambas se justifican a partir de una especie de regresión infinita de pruebas tras pruebas, de muestras, tras muestras. La verificación terminaba siendo una auto-trampa por parte del investigador, al seleccionar solo las pruebas y evidencias que favorecieran una hipótesis previa. Frente a esto, Popper propone una nueva fundamentación epistemológica a la ciencia, la lógica debe darse a partir del método hipotético deductivo como una metodología que parte de la formulación de la teoría para 
que sea contrastada vía refutación tanto en el plano lógico teórico como en el plano real empírico. Si una teoría resiste las críticas entonces queda corroborada momentáneamente (Popper. 1979, 1980). Este planteamiento fue ampliado por Lakatos al introducir la noción de programas de investigación. "El programa consiste en reglas metodológicas: algunas nos dicen las rutas de investigación que deben ser evitadas (heurística negativa) y otras, los caminos que deben seguirse (heurística positiva)". (Lakatos, 1998, 65). Este autor introduce el concepto de falsacionismo sofisticado, frente al falsacionismo ingenuo de Popper. Lakatos "sostiene que sustituye del concepto de teoría, como concepto básico de la lógica de la investigación, por el concepto de serie de teorías".

Estas nuevas formas de fundamentar la ciencia, (la probabilística, la del lenguaje y la de la contrastación), a pesar de su importancia llegaron a ocupar una posición secundaria en el ámbito institucional, al imponerse siempre el modelo empirista de verificación y cuantificación del conocimiento. El empirismo se consolidó como paradigma hegemónico en el mundo occidental, específicamente en los Estados Unidos al aliarse con la concepción pragmática de la realidad. Y América Latina se limitó institucionalmente a copiarlos.

\section{Thomas Kuhn y el progreso de la ciencia:}

Kuhn define paradigma de la siguiente manera: González (2004).

Considero a los paradigmas como realizaciones científicas universalmente reconocidas que, durante cierto tiempo, proporcionan modelos de problemas $\mathrm{y}$ soluciones a una comunidad científica.

Como en el caso de la ciencia, los conocimientos de la ingeniería se han organizado en disciplinas básicas con una estructura lógica semejante a la de aquellas teorías. Por ejemplo, la mecánica de los medios continuos, la teoría de circuitos eléctricos, la teoría de sistemas de control automático o la informática se exponen en libros que, en principio, en nada desmerecen de los que un científico consideraría aceptables y dignos de consideración.

Algunas de ellas, como las dos primeras, podrían figurar como capítulos de un libro de física general; y las dos segundas, de uno de matemáticas. No se olvide que la propia termodinámica está a caballo entre la física y la ingeniería; y aunque los físicos la reclaman como parte de su patrimonio, algunos la consideran una teoría fenomenológica —consideración en la que está implícita una calificación como de segunda fila. Por ello, en ciertos ámbitos, se habla de ciencias de la ingeniería.

Desde la física de Einstein y la mecánica cuántica, desde la teoría general de los sistemas planteada en la biología por Bertalanffi (1975), y la teoría de los sistemas cibernéticos, o desde científicos como Bohm (1998), Prigogine (1997), 
Morin (1997), Capra (1985, 1992, 1998) Chopra (1991), Luhmann (1998), Ferguson (1990) y otros intelectuales explican que la realidad es un sistema en permanente interacción y cambio. A nivel ontológico se admite el azar. Por ello éste puede conjugarse o complementarse con el orden. El progreso y la entropía se encuentran y son simultáneos. A pesar de la crisis, las contradicciones o el caos, el hombre puede generar estructuras superiores a las actuales, no siempre el caos genera más caos. Pueden emerger estructuras disipativas como lo plantea Prigogine, con una estructura superior y más compleja que la original. No hay un orden absoluto, los postulados del cambio, del devenir, de la existencia de varios órdenes, son los postulados que se aceptan. Debe quedar para la reflexión la controversia entre Einstein y Bohr, en el sentido en que el primero creía que Dios no jugaba a los dados con el universo.

Independientemente de las diferentes expresiones de la postmodernidad, que van desde un nihilismo hasta el ciberespacio, se tiene que reconocer que presenta, a nivel epistemológico, una ruptura con la modernidad, al admitir que la realidad es compleja, cambiante, incierta y turbulenta y que frente a ésta es necesario la comprensión de complejidad social o natural, así como la comunicación múltiple entre diferentes saberes culturales, para abordar los diferentes problemas. Aguilar (2018)

Los autores que han estado vinculados directa o indirectamente con el movimiento de la postmodernidad como Lyotard, Habermas, Derrida, Deleuze, Baudrillard, Lipovetsky, y Vattino al criticar la razón y la homogeneidad de la realidad, terminan admitiendo la necesidad de tener entonces una visión pluralista tanto de la misma realidad como del conocimiento en general y de la ciencia en particular.

\section{REFERENCIAS BIBLIOGRÁFICAS}

Aguilar Marín Pablo (2018). Epistemología. Unidad 1. Universidad Nacional del SantaEscuela de Postgrado.

Aguilar Marín Pablo (2018). Las primeras etapas del racionalismo y empirismo y del desarrollo de la ciencia. Unidad 2. Universidad Nacional del Santa - Escuela de Postgrado.

Aguilar Marín Pablo (2018). Enfoques lógico-positivista, hipotéticodeductivo, paradigmático y cibernético-sistémico de la ciencia. Unidad 3. Universidad Nacional del Santa - Escuela de Postgrado.

Castillo Ascanio Wilson Andrés (2015).

Estatuto epistemológico de la ingeniería Agroindustrial. Universidad Popular del Cesar (U.P.C).

Domínguez Castañeda Jorge Marino (2019). Director de la Escuela Profesional de Agroindustrial. Universidad Nacional del Santa. 
Felizzola Jiménez Heriberto y Luna Amaya Carmenza (2014). Lean Six Sigma en pequeñas y medianas empresas: un enfoque metodológico. Ingeniare. Revista chilena de ingeniería, vol. $22 \mathrm{~N}^{\circ} 2$, 2014, pp. 263-277.

González Fredy (2004). ¿Qué es un paradigma? Análisis teórico, conceptual y psicolingüístico del término. Investigación y Postgrado, Vol. $20 \mathrm{~N}^{\mathrm{o}} 1.2005$

Méndez Evaristo (2000). El desarrollo de la ciencia. "un enfoque epistemológico". Espacio Abierto, Vol. 9 - No. 4 / ISSN 1315-0006. octubre-diciembre 2000 / pp. 505-534.

Vejarano Mantilla Ricardo David (2015). UPN -Universidad Privada del Norte - Facultad de Ingeniería.

\section{CORRESPONDENCIA:}

Mag. Gino Paul Prieto Rosales

gpaulprieto@gmail.com 